issues remain unresolved. For instance, the cyclic stretch that LSECs undergo in vivo when the vessel widens after exposure to accelerated perfusion is biaxial - that is, the cell is stretched both along the direction of the vessel and sideways. By contrast, Lorenz and colleagues' ex vivo and in vitro experiments imparted only uniaxial cyclic stretch ${ }^{8}$. This difference might bias the signalling and angiocrine outputs the group observed. Whether other vascular mechanosensor receptors have a role in the induction of angiocrine factors also needs to be elucidated ${ }^{9}$.

In addition, the role of this biomechanically responsive pathway during injury remains to be dissected. Excessive increases in shear stress (for example, as a result of acute loss of liver mass) could be detrimental, leading to suboptimal liver regeneration. Lorenz et al. also did not directly assess whether lack of biomechanical activation of integrin $\beta 1$ and VEGFR3, as might occur in diseases such as diabetes, would lead to decreases in the liver's regenerative potential ${ }^{1,2}$.

In future, the ideal magnitude of cyclic stretch or shear stress required to initiate the physiological induction of angiocrine factors should be studied. The recruitment of circulating endothelial progenitor cells (EPCs), which are thought to supply the liver with HGF, could also be affected by shear-dependent activation of LSECs, further altering the liver's supply of angiocrine factors ${ }^{10}$. Indeed, how increased biomechanical forces alter the delivery of regenerative modulators to the liver, including circulating EPCs, inflammatory cells and platelets, to drive liver growth without encouraging scarring, needs further investigation.

Exactly how do integrin $\beta 1$ and VEGFR3 upregulate angiocrine factors? It is plausible that fluid shear stress induces integrin-mediated nuclear localization of specific transcription factors and so promotes the expression of angiocrine-factor genes ${ }^{2-4}$. Furthermore, integrin-mediated modulation of the elasticity of the extracellular matrix around hepatocytes in response to shear stress could also modulate hepatocyte proliferation. But what about VEGFR3? Proteins of the VEGFR family are activated by phosphorylation. Biomechanically independent phosphorylation of VEGFR2 on LSECs activates the protein $\mathrm{AKT}$, which recruits the transcription factor Id1 to DNA, inducing the expression of Wnt2 and $H G F$ genes ${ }^{2}$. But the mechanism by which phosphorylation of VEGFR3 turns on angiocrine factors is unknown.

These questions notwithstanding, Lorenz and colleagues' work takes into consideration the complexity of the biophysical environment to which LSECs are exposed in vivo, and so solves a mystery that has puzzled liver biologists for decades. The development of strategies that precisely regulate the magnitude of shear stress and cyclic stretch in the liver vasculature might restore angiocrine-dependent regenerative functions of the liver in pathological conditions, such as in cirrhosis, hepatitis and vascular abnormalities. This could in turn open the door to more-effective therapeutic liver regeneration. $\mathbf{-}$

Sina Y. Rabbany and Shahin Rafii are in the DeMatteis School of Engineering and Applied Science, Hofstra University, New York, New York 11548, USA, and in the Division of Regenerative Medicine, Ansary Stem Cell Institute, Weill Cornell Medicine, New York. e-mails: sina.y.rabbany@hofstra.edu; s.rafii@med.cornell.edu
1. Rafii, S., Butler, J. M. \& Ding, B.-S. Nature 529, 316-325 (2016).

2. Ding, B.-S. et al. Nature 468, 310-315 (2010).

3. Hu, J. et al. Science 343, 416-419 (2014).

4. Rocha, A. S. et al. Cell Rep. 13, 1757-1764 (2015).

5. Lorenz, L. et al. Nature 562, 128-132 (2018)

6. Rabbany, S. Y., Ding, B.-S., Larroche, C. \& Rafii, S. in Mechanical and Chemical Signaling in Angiogenesis (ed. Reinhart-King, C. A.) 19-45 (Springer, 2012).

7. Song, Z. et al. Semin. Cell Dev. Biol. 71, 153-167 (2017).

8. Wang, J. H.-C., Goldschmidt-Clermont, P., Wille, J. \& Yin, F. C.-P. J. Biomech. 34, 1563-1572 (2001).

9. Baeyens, N., Bandyopadhyay, C., Coon, B. G., Yun, S. \& Schwartz, M. A. J. Clin. Invest. 126, 821-828 (2016).

10.DeLeve, L. D. J. Clin. Invest. 123, 1861-1866 (2013).

This article was published online on 26 September 2018.

\title{
MICROBIOLOGY
}

\section{The electrifying energy of gut microbes}

Some bacteria make energy in a process that is accompanied by transfer of electrons to a mineral. A previously unknown electron-transfer pathway now reveals an energy-generation system used by bacteria in the human gut. SEE LETTER P.140

\section{LATY A. CAHOON \& NANCY E. FREITAG}

$\mathrm{T}$ he ability of certain bacteria to transfer electrons has been exploited for a variety of energy-generating applications, such as microbial fuel cells ${ }^{1}$, because the flow of charge carried by electrons underlies the process that generates electricity. It was thought that the capacity to achieve substantial levels of electron transfer occurred only in a specialized subset of bacteria. These microbes make energy by a mechanism that requires minerals for the electron-transfer process that accompanies energy generation ${ }^{2}$. On page 140, Light et al. ${ }^{3}$ report the discovery of an electrontransfer pathway in gut bacteria, and reveal that components of this pathway are present in diverse microbial species.

The molecule ATP provides the fundamental energy 'currency' for most cells, and is mainly produced by two mechanisms: fermentation, an anaerobic process in which ATP is generated from a limited repertoire of carbon sources, and respiration, a process that provides a high yield of ATP from a wide array of carbon sources and requires a compound that can accept electrons. In multicellular organisms, respiration involves electron transfer along an electron-transport chain that culminates in electrons being transferred to oxygen ${ }^{4}$.

By contrast, microbes can use a number of alternatives to oxygen as electron acceptors that enable respiration in anaerobic environments lacking fermentable energy sources ${ }^{2,5}$. For example, the bacteria Shewanella oneidensis and Geobacter metallireducens reside in mineral-rich environments, and these highly studied microbes have an anaerobic respiration process that uses minerals, such as iron(III) oxide $\left(\mathrm{Fe}_{2} \mathrm{O}_{3}\right)$, as respiratory electron acceptors ${ }^{2}$. However, because insoluble mineral deposits cannot be transported into the cell, mineral-respiring bacteria use a mecha$\mathrm{nism}^{2}$ called extracellular electron transfer (EET), in which electrons are transferred to the exterior of the cell. In the case of these bacteria, this process involves electron transfer from an NADH molecule to components that include a quinone molecule in the lipid membrane and a series of proteins containing haem groups that provide a path for electron transfer. The loss of an electron converts NADH to $\mathrm{NAD}^{+}$, which is used in the energy-generation process.

The food-borne bacterial pathogen Listeria monocytogenes sometimes has a host-associated part of its life cycle. This bacterium can infect humans, and can proliferate in nutrientrich environments that enable the use of fermentation as a metabolic strategy ${ }^{6}$. However, although $L$. monocytogenes has a life cycle in which neither minerals nor respiration is crucial for survival, Light et al. report that, when $L$. monocytogenes was placed in an electrochemical chamber in which an electrode can trap electrons, an electric current was generated, suggesting that this type of bacterium has the capacity for EET. This report now clarifies evidence presented decades ago ${ }^{7}$, indicating that this bacterium can change extracellular iron in the $\mathrm{Fe}^{3+}$ form to the $\mathrm{Fe}^{2+}$ 

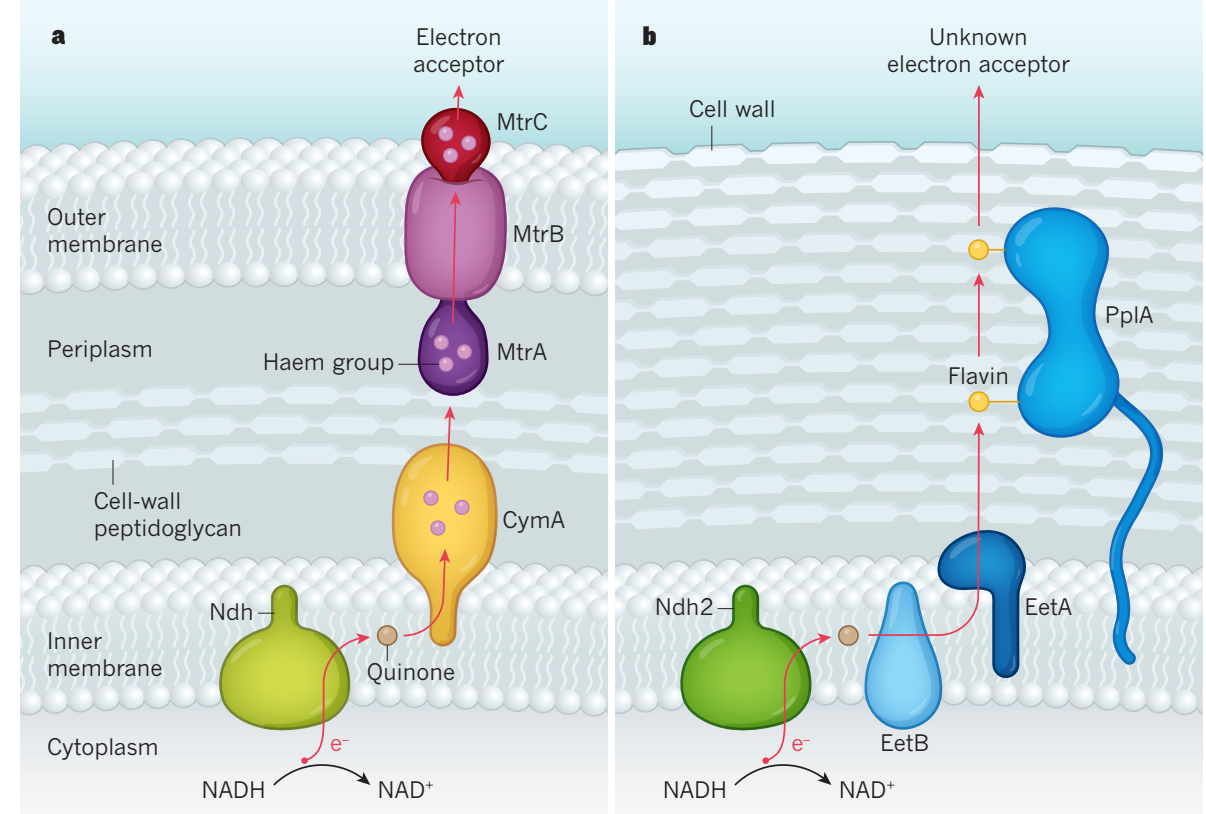

Figure 1 | Bacterial electron-transfer pathways. a, In the Gram-negative bacterium Shewanella oneidensis, the mechanism that generates energy from the molecule $\mathrm{NAD}^{+}$is accompanied by a process in which an electron ( $\left.\mathrm{e}^{-}\right)$from the molecule NADH is transferred outside the cell to a mineral such as iron(III) oxide that acts as an electron acceptor. In this process, known as extracellular electron transfer (EET), the electron-transfer path (red arrow) occurs across two lipid membranes and across the periplasm region, which contains cell-wall material that includes the sugar peptidoglycan. The electrontransfer route towards the cell exterior after crossing the protein Ndh includes a quinone molecule, haem groups associated with the proteins CymA, MtrA and MtrC, and transfer through the protein MtrB. b, Light et al. ${ }^{3}$ report a previously unknown EET mechanism in Listeria monocytogenes, a Gram-positive bacterium, which has only a single membrane. The authors identified components of this EET system, including the proteins Ndh2, EetB, EetA and PplA (which is associated with two flavin molecules). This newly identified EET process might occur in diverse bacteria, including those in the human gut. The electron acceptor for the pathway is unknown.

form, an alteration that might indicate electron transport out of the cell.

Using a combination of genetic and biochemical approaches, Light et al., true to the name, shed light on the molecular basis of this newly discovered form of EET. They identified the proteins Ndh2, EetB, EetA and PplA as being key components of this process. They show that the initial electron-transfer steps of EET in L. monocytogenes resemble those already known in mineral-respiring specialists. For example, electron transfer from the cell cytoplasm to a quinone molecule in the lipid membrane is similar to the steps of a conventional electron-transport chain. However, beyond this point, the mechanisms become more distinct. L. monocytogenes is a Grampositive bacterium, which means that it has a single lipid membrane and a thick cell wall. By contrast, S. oneidensis and G. metallireducens are Gram-negative bacteria, which have two lipid membranes separated by a region called the periplasm that contains cell-wall material. In these bacteria, tens of haem molecules bound to three types of protein establish a path for electrons to move across the periplasm and the outer lipid membrane ${ }^{8}$. By contrast, in L. monocytogenes, a single protein called PplA that contains two flavin molecules suffices to genus Lactobacillus.

This observation is intriguing because EET usually provides energy in anaerobic conditions, and growth strategies for such conditions can be important for microbial proliferation in the mammalian gut ${ }^{9}$. Indeed, Light et al. found that genes encoding components of the EET system they identified are required for L. monocytogenes to grow in anaerobic conditions. Moreover, when the authors monitored the ability of L. monocytogenes strains to colonize the mouse gut, the strains deficient in components of this EET system were at a competitive disadvantage, suggesting that EET has a key role in bacterial survival in this context. Investigating the role of EET in host-microbe interactions could offer an exciting direction for future research.

A central question raised by these findings is why EET might have evolved outside the context of mineral-respiring specialists. The bacterial environment may provide a clue. When microbes such as L. monocytogenes live in a host gut, they are immersed in nutrients, including flavin molecules, and Light et al. show that the presence of flavins potently enhances EET activity. The electron-transfer apparatus is simpler in Gram-positive bacteria than in Gram-negative bacteria. It stands to reason that an abundance of environmental flavins might produce a scenario in which evolution favours the minimal investment in protein infrastructure needed to enable EET in certain Gram-positive bacteria. EET might be used by certain mineral-respiring bacteria because it is crucial for their survival, whereas L. monocytogenes might use EET because it provides an opportunity to easily generate energy in certain environments.

The electron acceptor used by L. monocytogenes for EET is unknown. The bacterium might encounter conditions in which minerals represent an attractive electron acceptor, but it seems more probable that the highly reactive flavins in this pathway aid electron transfer to compounds such as organic soil components, disulfide groups on proteins or even other microbes ${ }^{10,11}$. If this is the case, in contrast to EET associated with specialized mineral respiration, the EET in L. monocytogenes might provide a more flexible mechanism for moving electrons to a variety of environmental acceptors.

It is a shock to the system to consider that microbes might be living highly charged lives in our gut. Light and colleagues' work provides a foundation for future investigation regarding such microbial existence. Furthermore, the characterization of this previously unknown EET mechanism might create opportunities for the design of bacteria-based energygenerating technologies.

\section{Laty A. Cahoon and Nancy E. Freitag} are in the Department of Microbiology and Immunology, University of Illinois at Chicago, Chicago, Illinois 60612, USA.

e-mail:nfreitag@uic.edu
1. Lovley, D. R. Annu. Rev. Microbiol. 66, 391-409 (2012).

2. Shi, L. et al. Nature Rev. Microbiol. 14, 651-662 (2016).

3. Light, S. H. et al. Nature 562, 140-144 (2018).

4. Richardson, D. J. Microbiology 146, 551-571 (2000).

5. Glasser, N. R., Saunders, S. H. \& Newman, D. K. Annu. Rev. Microbiol. 71, 731-751 (2017).

6. Freitag, N. E., Port, G. C. \& Miner, M. D. Nature Rev. Microbiol. 7, 623-628 (2009).

7. Deneer, H. G. \& Boychuk, I. Can. J. Microbiol. 39 480-485 (1993).

8. Nealson, K. H. \& Rowe, A. R. Microb. Biotechnol. 9, 595-600 (2016).

9. Winter, S. E. et al. Science 339, 708-711 (2013).

10. Scheller S., Yu, H., Chadwick G. L. McGlynn S. E. \& Orphan V. J. Science 351, 703-707 (2016).

11.Summers, Z. M. et al. Science 330, 1413-1415 (2010).

This article was published online on 12 September 2018. 